\title{
Data Mining In A Vibration Analysis Domain By Extracting Symbolic Rules From Rbf Neural Networks
}

\author{
Mohammad Amin Rashidifar*, Ali Amin Rashidifar ${ }^{* *}$, Darvish Ahmadi* \\ *Department of Mechanical Engineering, Islamic Azad University, Shadegan Branch, Shadegan, Iran \\ ${ }^{* *}$ Department of Computer Science, Islamic Azad University, Shadegan Branch, Shadegan, Iran
}

\begin{tabular}{|c|c|}
\hline Article Info & ABSTRACT \\
\hline Article history: & Neural networks are becoming an increasingly popular technique for \\
\hline Received Jun $12^{\text {th }} 2015$ & modelling data with complex and/or non-linear relationships. Diagnostic \\
\hline Revised Aug $20^{\text {th }}, 2015$ & category, especially those using spectral vibration data. However, neural \\
\hline Accepted Aug $26^{\text {th }}, 2015$ & $\begin{array}{l}\text { networks do have some major disadvantages compared with rule based } \\
\text { diagnostic systems. The most important criticism is the lack of any }\end{array}$ \\
\hline Keyword: & $\begin{array}{l}\text { explanation system, which would open up the neural networks internal } \\
\text { operation for scrutiny. This paper illustrates how the internal parameters of }\end{array}$ \\
\hline Neural network & $\begin{array}{l}\text { an RBF network can be converted into symbolic rule format. The rule } \\
\text { extraction algorithm and is described in detail. }\end{array}$ \\
\hline
\end{tabular}

Rule extraction

knowledge discovery

Copyright $@ 2016$ Institute of Advanced Engineering and Science. All rights reserved.

\section{Corresponding Author:}

Mohammad Amin Rashidifar,

Department of Mechanical Engineering,

Islamic Azad University, Shadegan Branch.

Email: rashidifar_58@yahoo.com

\section{INTRODUCTION}

Neural networks have been applied to many real-world, large-scale problems of considerable complexity. They are useful for pattern recognition and they are robust classifiers, with the ability to generalise in making decisions about imprecise input data (Bishop95). They offer robust solutions to a variety of classification problems such as speech, character and signal recognition, as well as functional prediction and system modelling where the physical processes are not understood or are highly non-linear. Although neural networks have gained acceptance in many industrial and scientific fields they have not been widely used by practitioners of mission critical applications such as those engaged in aerospace, military and medical systems. This is understandable since neural networks do not lend themselves to the normal software engineering development process. Knowledge extraction by forming symbolic rules from the internal parameters of neural networks is now becoming an accepted technique for overcoming some of their limitations. In this paper we describe our method of extracting knowledge from an RBF network which is classed as a local type of neural network.

\section{KNOWLEDGE EXTRACTION FROM NEURAL NETWORKS}

In recent years there has been a great deal of interest in researching techniques for extracting symbolic rules from neural networks. Rule extraction has been carried out upon a variety of neural network types such as multi-layer perceptrons, Kohonen Networks, recurrent networks (Andrews-et-al). The advantages of extracting rules from neural networks can be summarised as follows: 
a. The knowledge learned by a neural network is generally difficult to understand by humans. The provision of a mechanism that can interpret the network input/output mappings in the form of rules would be very useful.

b. Deficiencies in the original training set may be identified, thus the generalisation of the network may be improved by the Addition/enhancement of new classes. The identification of superfluous network parameters for removal would also enhance network performance.

c. Analysis of previously unknown relationships in the data.This feature has a huge potential for knowledge discovery/data mining and possibilities may exist for scientific induction.

In addition to providing an explanation facility, rule extraction is recognised as a powerful technique for neuro-symbolic integration within hybrid systems (McGarry99a).

\section{RADIAL BASIS FUNCTION NETWORKS}

Radial basis function (RBF) neural networks are a model that has functional similarities found in many biological neurons. In biological nervous systems certain cells are responsive to a narrow range of input stimuli. Figure 1 shows a network trained the $\mathrm{X}$ or data set for illustration. This network has two input features, two output classes and four hidden units.

The RBF network consists of a feed forward architecture with an input layer, a hidden layer of RBF "pattern" units and an output layer of linear units. The input layer simply transfers the input vector to the hidden units, which form a localised response to the input pattern. Learning is normally undertaken as a twostage process.

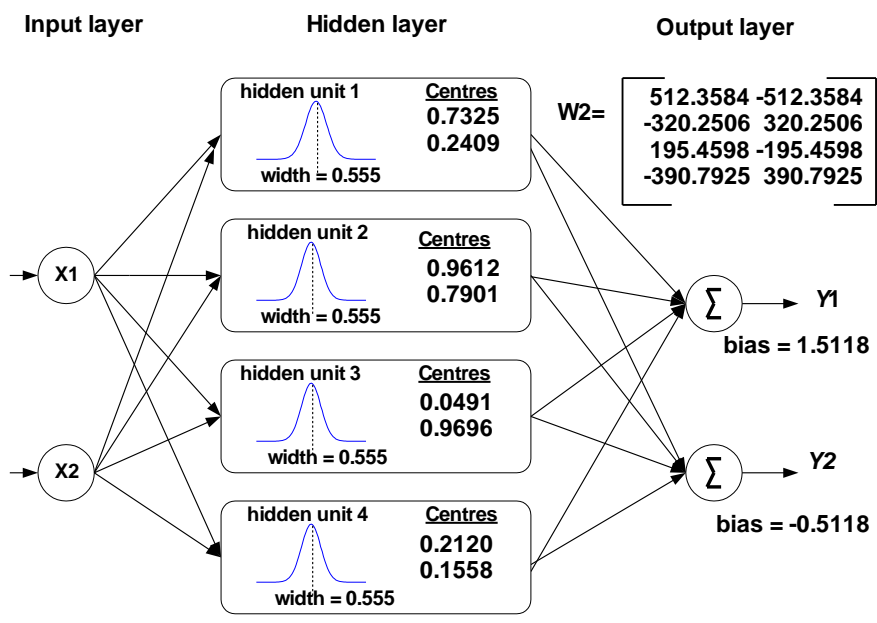

Figure 1. RBF network

\subsection{RBF Training}

The accuracy levels stated in the tables are the best out of up to 10 test runs. Training of the RBF networks required the setting of three parameters, the global error, the spread or width of the basis function and the maximum number of hidden units. The value assigned to the global error setting can result in fewer hidden units being used than the maximum value. If the error value is not reached, training would terminate when the maximum number of hidden units has been assigned. The training and test data for the construction of the RBF networks were generally split 50/50.

\subsection{Data Sets}

The data used in this work was created by the VISION project which was funded by the Brite EuRam initiative of the European Union (Project No. BE95-1313)

a. Vibration data A. This is a synthetic data set composed of simulated data generated by finite element modelling (FEM). The data was gathered as part of the VISION project in which the University of Sunderland was a key partner. The data is composed of 1028 examples with nine input features and three output classes. The nine input features represent vibration spectra that are indicative of the 
physical operating condition of a particular type of rotating machine such as a motor or fan. The three output classes are composed of two faults and the normal operating condition.

Vibration data B. This is a more complex data set produced by the VISION project and is a combination of real world data, simulated FEM data and test rig data. This data is a subset of that used to produce the final diagnostic model incorporated into end product. It consists of 1862 examples with 20 input features and eight output classes (McGarry99b). Table 1 displays a list of FFT parameters with selected faults in the SIMU column.

Table 1. Statistical Parameter Number Identifier

\begin{tabular}{rlll}
\hline PAR & SIMU & RESPFFT & STATIST \\
\hline 1 & TTime & FFRPM & SRms \\
2 & AFreRange & FF2RPM & SStanDevi \\
3 & ATimePoints & FF3RPM & SAverage \\
4 & AWindow & FF4RPM & SMeanDevi \\
5 & AAveDomain & FF5RPM & SMaximum \\
6 & AAveNum & FFHarmPow & SMinimum \\
7 & CRPM & FF10-1000 & SKurtosis \\
8 & CPower & FF500-1000 & SSkewness \\
9 & GBearing & FF1000-2000 & SCrestFactor \\
10 & GShaft & FF312-1200 & Si_Rms \\
11 & GSupport & FF1200-10000 & Si_StanDevi \\
12 & FNoise & FFRMS & Si_Average \\
13 & FUnbalance & FFMaximum & Si_MeanDevi \\
14 & FMiss & FFMaximumFreq & Si_Maximum \\
\hline
\end{tabular}

\section{DATA MINING AND KNOWLEDGE DISCOVERY}

Over the last 10 years data mining has aroused a great deal of interest as a solution to the problems associated with searching for informative patterns in large data bases. Artificial intelligence techniques can provide the means to build automated, adaptive systems. Data mining (DM) is a process concerned with the semi-automatic extraction of interesting patterns from databases. The search for interesting and potentially useful knowledge requires the definition of what makes an interesting pattern? This is the most important question concerning DM as it is all too easy for the DM process to produce large quantities of patterns, which can overwhelm the user. Many patterns will be either: too regular and therefore trivial or non-informative, or too random and therefore meaningless. Over the years much research has been carried out on the "interestingness" problem (Piatetsky94). The actual data mining process may involve a number of artificial intelligence techniques. Some techniques which have been successfully used in the past are decision trees, inductive logic programming, genetic algorithms, neural network and case based reasoning.

\subsection{Data Mining for Condition Monitoring Applications}

Over the years vibration consultants have amassed huge databases of spectral information. This information is usually taken during routine analysis of machinery and the diagnosis may be conducted on site but more usually back at the consultant's premises. Hence the requirement for mass storage and a comprehensive database archive system. In addition to the storage of the actual vibration data details such as customer site, type and location of the machine, the measurement locations and types of transducer used may be kept. It is possible to identify several sources of new knowledge that may reside within the accumulated data store:

a. Discovering new machine faults. The likelihood of discovering some new configuration of mechanical defect is most unlikely considering the relative maturity of the electro-mechanical field. However, it is possible for a new product such as a pump or a bearing assembly to suffer from a design error that may exhibit an unusual frequency signature during operation.

b. Discovering new features to identify known machine faults. This is potentially the most useful area for data mining and knowledge discovery. In recent years a great deal of research effort has been expended in an attempt to discover those frequency components that lead to an accurate diagnosis of machine condition. It is evident that a great deal of diagnostic knowledge still remains to be discovered.

c. Discovering the cause of spurious diagnosis. The ability to detect and discover the cause of an incorrect diagnosis will greatly improve the reliability of a diagnostic system. Currently this feature is very difficult to implement, as this requires the intervention of a human operator to detect the incorrect diagnosis and some further analysis of the rogue input data. Usually, a precise explanation of why any given input data was incorrectly diagnosed as a fault is impossible. 
The data mining technique described in this paper involves the process of training several RBF networks on vibration data and then extracting symbolic rules from these networks as an aid to understanding: i) RBF network internal operation. ii) The relationships of the parameters associated with each fault class.

\section{LREX: LOCAL RULE EXTRACTION ALGORITHM}

The development of the LREX (Local) algorithm was motivated by the local architecture of RBF networks, which suggested that rules with unique characteristics could be extracted. The basic premise of our rule extraction process works on the assumption that each hidden RBF unit can be uniquely assigned to a specific class. The LREX algorithm is composed of two modules: the mREX module extracts IF...THEN type rules based on the premise that a hidden unit can be uniquely assigned to a specific output class. However, hidden unit sharing occurs within networks trained on non-linear or complex data. This phenomenon reduces rule accuracy as several hidden units may be shared amongst several classes. The second module, hREX was developed to identify which hidden units are shared between several classes. The extracted rules are IF...THEN type rules hidden may appear and details are provided elsewhere in the literature (Mcgarry01a).

\section{1. mREX: Input-to-output mapping}

The functionality of mREX algorithm is shown in figure 2. The first stage of the mREX algorithm is to use the W2 weight matrix (see Figure 1) to identify the class allocation of each hidden unit. The next stage is to calculate the lower and upper bounds of each antecedent by adjusting the centre weights using the Gaussian spread. The lower and upper limits are further adjusted using a statistical measure $\boldsymbol{S}$ gained.

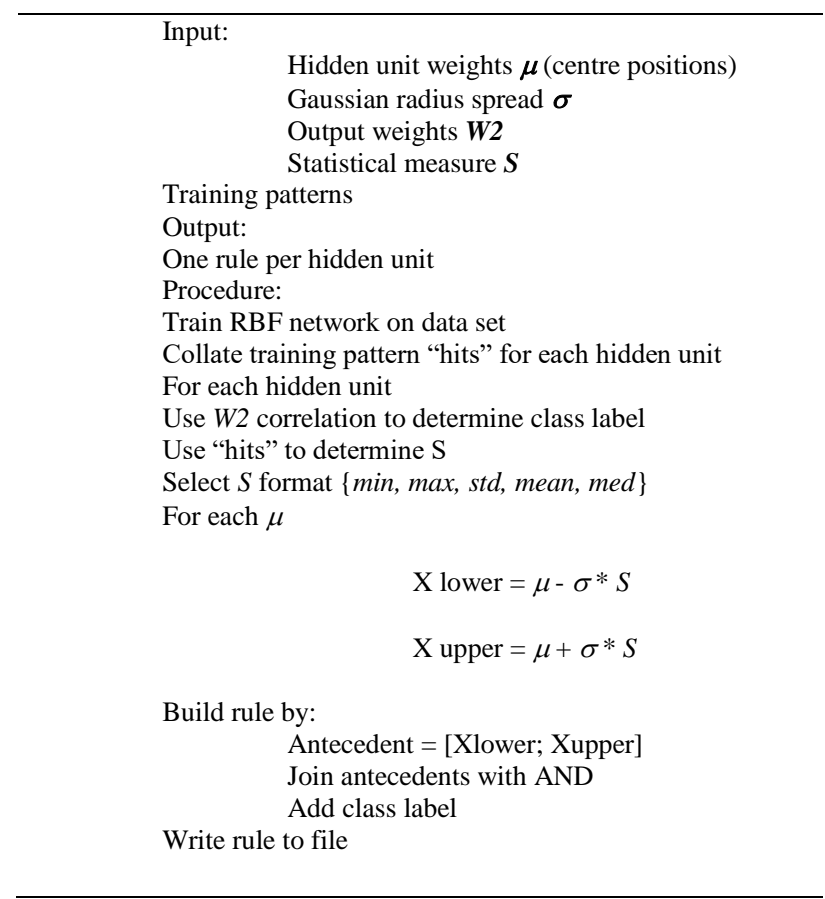

Figure 2. mREX Rule Extraction Algorithm

From the training patterns classified by each hidden unit. $S$ is used empirically to either contract or expand each antecedent's range in relation to the particular characteristics of these training patterns. A single rule from the vibration a domain is presented in Figure 3. 


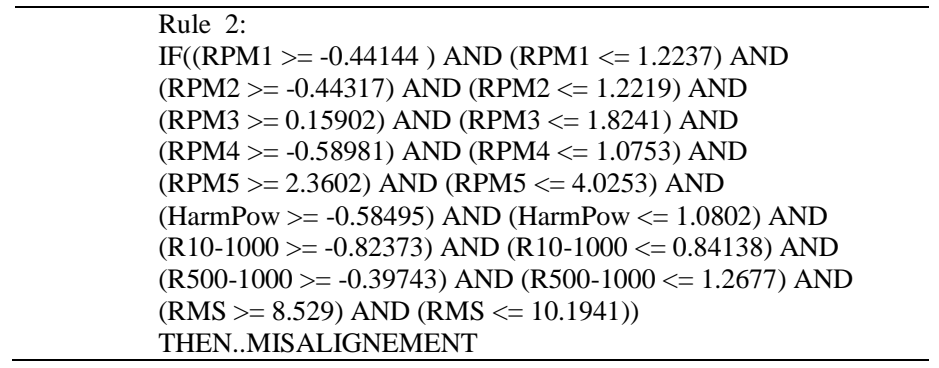

Figure 3. mREX extracted rule for misalignment (Vibration A data).

\subsection{Enhancements to the Extracted Rules}

A number of features were added as a postprocessing function to the rule extraction algorithm. Although these additions are not directly a part of rule extraction they do increase the understandibility of the extracted rules and therefore add value to the overall process of data mining: removal of superfluous antecedents, removal of redundant rules and the addition of a default rule. A fragment of the rule set for the vibration a domain is presented in Figure 4.

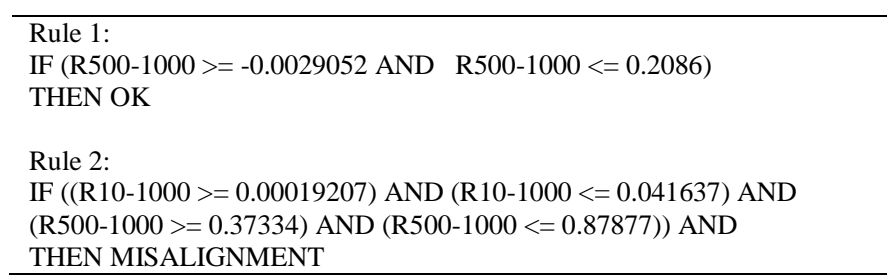

Figure 4. Extracted Rules From The Vibration a Domain with Superfluous Rules Removed.

The use of information theory was made to reduce the number of antecedents within a rule. The information measure or information gain about an input feature represents the importance of that feature to resolve class identity. Features with very low values may be excluded from the extracted rules and will thus simplify the rule and aid comprehensibility. Removal of redundant rules was accomplished by identifying those rules which only classified a small number (1-2) of examples. A small amount of accuracy was lost but reduced the rule set by 25 rules in the case of the vibration a data. The number of redundant rules varied with the size of the original RBF network and reflects the inefficiency of the training algorithm used. The addition of a default rule improved the accuracy of rules from vibration data A. This is always a dangerous strategy as the rules are forced to make a classification decision regarding the input i.e. the class with the largest number of rules had all of its rules removed, the remaining rules would check for the other classes and if these failed then the default class would be selected. The accuracy results of the basic and three rule type variants are presented in Table 2 .

Table 2. Accuracy comparison between original RBF network, basic rule, pruned antecedent, pruned rule and default rule systems.

\begin{tabular}{llllll}
\hline Data set & RBF $(\%)$ & Basic rule (\%) & Ante-pruned (\%) & Rule pruned (\%) & Default rule (\%) \\
\hline Vibration A & 89 & 73 & 50 & $65: 25$ & 70 \\
Vibration B & 94 & 80 & 45 & $80: 6$ & 84 \\
\hline
\end{tabular}

Analysis of the hREX rules proved to be interesting as the RBF networks have up to $20-40 \%$ of their hidden units shared between the various output classes. Overall, pruning rules with "superfluous" antecedents produced a very poor result. The rating assigned to each antecedent (input feature) was calculated on an individual basis and did not include inter-variable relationships. However, further inspection of those rules that had antecedents pruned but had good accuracy highlighted some interesting details. It would appear that the usual FFT parameters (i.e. the RPM's) for determining fault type and severity appear not to be required by 
several rules. These rules seem to have modelled facets of the original data set learned by the RBF network. However, the rules are valid, accurate and account for $15-20 \%$ of the total number of extracted rules.

\section{CONCLUSIONS}

The work described in this paper has tackled the issue of rule extraction from RBF networks. The rules provide the user with lower and upper bounds for each parameter associated with a particular class of fault. Deficiencies in the original RBF were identified by the removal of those rules (i.e. hidden units) that either do not contribute or contribute little to the overall classification capability. The removal of superfluous antecedents enables a smaller sized rule to be constructed and thus aids comprehensibility. This technique has also identified rules that use parameters not typically associated with fault diagnosis and thus has aided a better understanding of the data set. Future work will examine other methods for antecedent removal that will assess the importance of inter-variable relationships instead of the information measure currently used which examines the importance of each variable individually.

\section{REFERENCES}

[1] Andrews et al, (1995) Andrews, R., Diederich, J., Tickle, AB., A Survey and Critique of Techniques for Extracting Rules from Trained Artificial Neural Networks. Knowledge-Based Systems, Vol.8, No6, pp373-389, 1995.

[2] Bishop, C. (1995), Neural Networks for Pattern Recognition, Oxford University Press.

[3] McGarry, K. and MacIntyre, J. (1999a), Hybrid diagnostic system based upon simulation and artificial intelligence, in "Proceedings of the International Conference on Condition Monitoring", University of Wales, Swansea, 593600.

[4] McGarry, K., Wermter, S. and MacIntyre, J. (1999b), "Hybrid neural systems: from simple coupling to fully integrated neural networks", Neural Computing Surveys 2:1, 62-93.

[5] McGarry, K., Wermter, S. and MacIntyre, J. (2001), Knowledge extraction from local function networks, in "Seventeenth International Joint Conference on Artificial Intelligence", Seattle, USA.

[6] Piatetsky-Shapiro, G. and Matheus, C. J. (1994), the interestingness of deviations, "Proceedings of AAAI Workshop on Knowledge Discovery in Databases". 